\title{
Vaccines against papillomavirus infections and disease
}

\author{
Luisa L. Villa
}

S quamous cell carcinoma of the uterine cervix is the second cause of cancer-related deaths in women, the higher incidence being observed in developing countries. Infection with oncogenic types of human papillomavirus (HPV) is considered the major risk factor for the development of malignancies in the uterine cervix. However, HPV is considered to be a necessary but not sufficient cause for cervical cancer and, therefore, other factors contribute to the carcinogenic process, both present in the environment and from the host.

Papillomaviruses are epitheliotropic viruses present in the skin and mucosa of several animals. In humans, more than 100 types have been described. Mucosal and genital HPVs, consisting of about 30 types, are divided into low-risk (HPVs 6, 11, 40, 42, 43, 44, 54, 61, 70, 72, 81) and high-risk (HPVs 16, 18, 31, 33, 35, 39, 45, $51,52,56,58,66,73$, and 82 ), according to their presence in malignant lesions of the cervix. The latter types have been adequately evaluated as high risk types in relation to invasive cervical cancer. For all of them, risk estimates were greater than 30 (range 35-350) strongly suggesting that these associations are causal in nature ${ }^{1,2}$.

The genome of these viruses is a double stranded DNA molecule of about 8000 base pairs, with three identified regions: a late region $(\mathrm{L})$ containing 2 genes -L1 and L2- which encode the viral capsid proteins; an early region (E) encoding for proteins involved in viral DNA replication and control of viral transcription, such as E1 and E2, as well as the main transforming genes E6, E7 and E5; a long control region (LCR), containing several binding sites for nuclear and viral transcriptional factors as well as promotor sequences, is found between the L and E regions. HPV genomes are found as episomes in the nucleus of infected cells of the normal cervix, where infective viral particles can be isolated. However, in some low-grade and in most of the high-grade lesions of the uterine cervix, including cancer, HPV genomes are found integrated into the host genome. A disruption of the E1-E2 region is required for HPV genome integration. This event results in an increased expression and stabilization of the E6 and E7 transcripts. The E6 protein from highrisk HPVs binds cellular p53, promoting the degradation of $\mathrm{p} 53$ by the cellular ubiquitin proteolysis system.
The E7 protein interacts with $\mathrm{pRB}$ and inactivates this cellular protein. As a consequence, E2F transcription factor is released from pRB-E2F complex, leading to transcriptional activation of several genes involved in cell proliferation. Such interactions of HPV E6 and E7 proteins interfere with several pathways involved in the control of the cell cycle and DNA repair ${ }^{3}$.

Infection with high-risk HPV types is frequent among sexually active women, with incidence ranging from 15 to $40 \%$. When additional cervical specimens are taken from these women in follow-up surveys, the majority of the infections are found to be transient. However, a small proportion of infected women have persistent infection with high-risk HPV types. Previous reports have demonstrated that women persistently infected with oncogenic HPV types are more likely to develop malignant cervical lesions.

Infections with HPV are accompanied by an immune response that is both humoral and cellular ${ }^{4}$. Several studies have shown that serological diagnosis of HPV infection using genetically engineered HPV capsids (also known as Virus-Like Particles or VLP) correlate well with HPV DNA presence in cervical smears. The antibodies invoked recognize type-specific conformational epitopes present on VLPs, particularly against the viral capsid protein L1, and the humoral response (IgG) against HPVs is stable over time. Neutralizing antibodies are generated, although frequently in low titers, and are considered to be host protective against further infection with that virus type. Therefore, VLP serology has been used as a marker of cumulative exposure to HPV, and sexual behavior, despite the observation that seroconversion may be delayed or never occur in a subset of women testing positive for HPV DNA. Overall, frequency and titer of several types of antibodies generated against HPV show a great variability that is dependent on the HPV type specificity, on the recognized epitopes, on the type of samples, and sensitivity of the assay. The mere definition of cutoff values can hinder comparison between different epidemiological surveys. Concerning type specificity, it has been demonstrated that VLPs of each HPV type induce serum antibody response that are genotype-specific with the exception of HPV types 6 and 11 which are considered to be cross-reactive and
Ludwig Institute for Cancer Research, Sao Paulo Branch São Paulo, SP, Brazil

Received: august $10^{\text {th }} 2005$ Acepted: august $24^{\text {th }} 2005$

Correspondence to: Luisa Lina Villa Ilvilla@ludwig.org.br 
HPV 31 and 45 who show low levels of cross-reactive antibodies against HPV 33 and 18, respectively. Likewise, variants of HPV 16 are considered to belong to the same serotype.

\section{Development of papillomavirus vaccines. Animal models}

Since the early 1990s, several groups have succeeded in generating recombinant L1 only or L1 and L2 virus-like particles (VLPs) from any papillomavirus known, that resemble very closely the native virions but are devoided of the viral genome. VLPs produced in both prokaryotic (bacteria) and eukaryotic cells (yeast, insect, mammalian) reproduce the antigenicity of native virions by presenting conformational epitopes that have been shown to induce type specific virus neutralizing antibodies. Although VLPs are the natural candidates for immunogens, several other antigens, including structural and nonstructural papillomavirus proteins have been tested in different protocols. In addition, HPV early proteins such as E7, E6 and E2 have been fused to VLPs. These chimeric VLP vaccines have been shown to induce neutralizing antibodies and to elicit CTL responses specific to the early viral protein in murine models, as mentioned below. Finally, recombinant viruses, in different vectors, have been used as DNA vaccines, either alone or in combination with adjuvants.

The animal models of papillomavirus infection have proved extremely valuable in the search and development of anti-viral vaccines both prophylactically and therapeutically. Immunization with purified speciesspecific VLPs induces neutralizing antibodies that protect against live virus challenge in rabbits, cows and dogs. Among these models, the mucosal infections caused by COPV (canine oral papillomavirus) and ROPV (rabbit oral papillomavirus) are those that resemble more the human genital papillomavirus infections. Interestingly, in bovines, vaccines based on the same viral target can be effective either prophylactically or therapeutically, inducing regression of early lesions. The duration of protection is longlasting, at least in rabbits where neutralizing antibodies have been induced upon injection of CRPV VLPs. In Rhesus macaques, vaccination with an HPV-16 L1 VLP vaccine induced strong humoral, including neutralizing antibodies, and cellular immune responses. Although no papillomavirus is known to infect the regular laboratory mice, experimental models are available based on mouse cell lines transformed with high-risk HPV capable of inducing tumors and metastases. Mouse models have been particularly useful in testing different administration routes: Intranasal and oral administration of HPV VLPs alone or in combination with different adjuvants have been shown to be immunogenic. Recently, HPV 16 L1 capsomeres, administered both sub-cutaneously and intranasally, have been shown to induce potent CTL responses, comparable to that obtained with VLPs. Similar results have been previously descrived for COPV-induced lesions in dogs. Murine models have been instrumental to show that anti-specific HPV responses can be obtained by DNA immunization, which can both control tumor and metastasis development and promote tumor regression. Altogether, the encouraging results obtained with different animal models have warranted the development of HPV vaccines in humans.

\section{Vaccines to prevent human papillomavirus infection}

Vaccines to prevent HPV infection aim to induce neutralizing antibodies directed to conformational epitopes of capsid proteins, which most probably will be type specific. The protection against infection depends on the amount of antibodies produced by the host, its availability at the infection site and the persistence of the neutralizing antibodies along time. Analogous to the studies conducted with animals, VLPs for the most prevalent HPV types have been generated and tested as vaccine candidates in several ongoing clinical trials. Most of these studies are randomized, placebo-controlled, blinded trials, which include young woman living in different countries around the World. So far, the published studies with prophylactic HPV vaccines in humans have shown that the vaccine is well-tolerated, generates strong immune responses that are several orders of magnitude higher than those exhibited by naturally infected populations, and prevent both infection and disease with high efficacies ${ }^{5}$. The first proof-of-principle study with a HPV 16 L1 VLP vaccine showed that women injected with HPV type 16 virus-like particles (VLPs) have been protected against infection when compared to the placebo group ${ }^{6}$. Moreover, during the 17-month period of follow-up, the few cases of cervical lesions attributed to this HPV developed only in women that did not receive the vaccine. The rate of efficacy of this vaccine was $90-100 \%$. These results are very encouraging and constitute the proof-of-principle of the feasibility of this monovalent HPV vaccine developed by Merck, Sharp and Dohme. However, HPV-16 accounts for about $50 \%$ of all cervical cancers, and as mentioned earlier, the immune responses against HPV are type specific. Therefore, efforts were made to 
develop multivalent vaccines that could control for most HPV infections associated with cervical disease namely HPV-18, -31, -33, -45, among other types. Moreover, low-risk HPV types are responsible for more than $90 \%$ of genital warts and recurrent respiratory papillomatosis. One of these studies is testing the safety and efficacy of a quadrivalent (HPV 6, 11, 16, 18) VLP vaccine. The study conducted in Brazil, USA, and several Nordic countries, followed more than 1,100 women for 30 months, and demonstrated that a quadrivalent HPV 6/11/16/18 vaccine based on yeastproduced L1 VLPs, containing aluminum hydroxide as adjuvant, induced high-titer anti-HPV serum antibody levels. Immune responses were sustained after 30 months of follow-up and remained at or above the level of antibodies developed in naturally exposed women $^{7}$. Importantly, this quadrivalent $6,11,16,18$ HPV VLP vaccine effectively prevented acquisition of infection and clinical disease caused by common HPV types, including genital warts and cervical intraepithelial neoplasia. Large-scale studies encompassing up to 25,000 young women are underway. Results from these phase III trials are expected to be available soon, so that the vaccine may be commercially available as early as 2006-2007. Further studies involve vaccination in adolescents, mid-adult Women and Men. A similar trial of a bivalent (16/18) HPV VLP vaccine formulated with AS04 adjuvant, sponsored by Glaxo SmithKline, demonstrated that this vaccine is about $90 \%$ efficacious in preventing persistent infections and associated cervical abnormalities in young women over a 27 month period $^{8}$.

\section{Therapeutic vaccines}

New therapeutic approaches to cervical cancer include immunization with early proteins and derived peptides of high-risk HPV, aiming to eliminate epithelial cells in the anogenital tract that are already infected with papillomaviruses. Natural immune responses to papillomavirus encoded antigens are weak, with the exception of the E7 protein, to which a humoral immune response is observed in most cases of invasive cervical carcinoma. Immune response to the E2 and E6 proteins may also predict the regression of papillomavirus-associated disease. Thus, targeting immunotherapy to some or all of the HPV early proteins, particularly E6 and E7, is being considered for treatment of papillomavirus-induced neoplasia. Immunogens include synthetic peptides, recombinant proteins, chimeric VLPs and live viral expression vectors. Preclinical studies in animal models indicate that some of these vaccines can eradicate HPV positive tumors.

Although it has been possible to demonstrate that several papillomavirus early proteins are immunogenic in patients with HPV-associated cervical cancer, clinical trials of therapeutic vaccines have been conducted in patients with advanced stage of disease in whom a poor immune response is expected. Nevertheless, some positive results are available and partial responses have been obtained in patients with high-grade intraepithelial lesions. Promising results have been obtained in patients with genital warts. Clinical trials are underway to demonstrate the immunogenicity of a therapeutic DNA vaccine for anal dysplasia, consisting of an encapsulated plasmid bearing the sequences of HPV-16 E7 and multiple HLA-A2-restricted epitopes. The potential use of dendritic cells carrying HPV antigens is under study.

Despite the encouraging results from several ongoing vaccine trials showing good safety profiles and induction of high titres of virus specific antibody by VLP based Papillomavirus vaccines, there are several open issues that require attention. Even if a vaccine is shown to be effective against a few HPV types, it is not known how long the protection will last and what will be the impact of this type of prevention in the natural history of cervical cancer. It is expected that a multivalent HPV vaccine will reduce the number of interventions such as colposcopies, biopsies and treatment of precursor lesions. However, Papanicolaoubased screening procedures, including the recently approved HPV DNA test for primary screening of cervical cancer in the U.S.A., will continue to play an essential role in screening for cervical cancer. Furthermore, we do not know if effective coverage against some genotypes could favor the emergence of more pathogenic types. Existence of tumor immune escape mechanisms puts therapeutic vaccines for HPV associated cancers in the same road as therapeutic vaccines for other cancers: so far, none have been shown to be completely effective. Development of multimodality treatments to induce strong immune responses and avoid escape from individual treatments are likely to contribute to the control of the precursors of cervical neoplasia of the uterine cervix and ultimately to invasive carcinoma.

References on page 163. (Spanish version). 


\title{
Vacunas contra infección y enfermedad causadas por papilomavirus
}

\author{
Luisa L. Villa
}

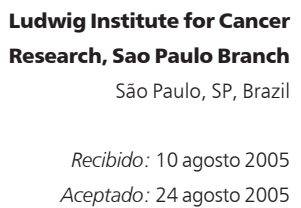

Correspondencia a: Luisa Lina Villa Ilvilla@ludwig.org.br
$\mathrm{E}$ 1 carcinoma de células escamosas de cuello uterino es la segunda causa de muerte relacionada a cáncer en mujeres, su mayor incidencia se observa en países en desarrollo. La infección con serotipos oncogénicos de papilomavirus humano (VPH) es considerada el factor de riesgo principal para el desarrollo de tumores malignos en el cuello uterino. Sin embargo, el VPH es una condición necesaria, pero insuficiente, para causar el cáncer cervical y otros factores contribuyen al proceso carcinogénico, presentes tanto en el medioambiente como en el hospedero.

Los papilomavirus son virus epitelio-trópicos presentes en la piel y en mucosas de diversos animales. En humanos, más de 100 serotipos han sido descritos. Los VPH de mucosas y genitales, alrededor de 30 serotipos, se dividen en de bajo riesgo (VPH 6, 11, 40, $42,43,44,54,61,70,72,81$ ) y de alto riesgo (VPH 16,18 , $31,33,35,39,45,51,52,56,58,66,73$, y 82 ), de acuerdo a su presencia en lesiones malignas del cérvix. Estos últimos serotipos han sido debidamente evaluados como serotipos de alto riesgo para el desarrollo de cáncer cervical invasor; para todos ellos, las estimaciones de riesgo superaron a 30 (rango 35-350 veces) sugiriendo fuertemente su relación causal en la naturaleza ${ }^{1,2}$

El genoma de estos virus es una molécula de ADN de doble cadena de cerca de 8.000 pares de bases, con tres regiones identificadas: una región tardía (late L) conteniendo dos genes -L1 y L2- la que codifica las proteínas de la cápside viral; una región temprana (early E) que codifica proteínas involucradas en la replicación del ADN viral y control de la transcripción viral, tal como E1 y E2, así como los principales genes de transformación E6, E7 y E5; entre las regiones L y E se encuentra una región larga de control (long control region $\mathrm{LCR}$ ), que contiene diversos sitios de unión para la factores de transcripción nuclear y viral, así como secuencias promotoras. En el núcleo de células infectadas del cérvix normal se encuentran genomas de VPH en estado episomal, desde donde pueden aislarse partículas virales infectantes. Sin embargo, en algunas lesiones de bajo grado y en la mayoría de las de alto grado del cuello uterino, incluyendo el cáncer, se encuentra genoma de VPH integrado en el genoma del hospedero. Se requiere una disrupción de las regiones E1-E2 para la integración del genoma de VPH. Este evento se traduce en una expresión aumentada y estabilización de los transcriptos de E6 y E7. La proteína E6 de VPHs de alto riesgo se liga a la proteína celular p53, promoviendo la degradación de p53 por el sistema proteolítico celular ubiquitina. La proteína E7 interactúa con $\mathrm{pRB}$ e inactiva esta proteína celular. Como consequencia, el factor de transcripción E2F es liberado del complejo pRB-E2F, llevando a la activación transcripcional de diversos genes involucrados en la proliferación celular. Tales interacciones de las proteínas E6 y E7 de VPH interfieren con diversas vías implicadas en el control del ciclo celular y reparación del $\mathrm{ADN}^{3}$.

La infección con serotipos de VPH de alto riesgo es frecuente entre las mujeres sexualmente activas, con una incidencia que oscila entre 5 y $40 \%$. Cuando se obtienen muestras cervicales adicionales en estudios de seguimiento de estas mujeres, la mayoría de las infecciones resultan ser transitorias. No obstante, una pequeña fracción de las mujeres infectadas tiene una infección persistente con serotipos de VPH de alto riesgo. Reportes previos han demostrado que mujeres persistentemente infectadas con serotipos oncogénicos de VPH tienen mayor probabilidad de desarrollar lesiones cervicales malignas.

La infección con VPH se acompaña de una respuesta inmune tanto humoral como celular ${ }^{4}$. Diversos estudios han comprobado que el diagnóstico serológico de infección por VPH usando cápsides de VPH diseñadas genéticamente (también denominadas las partículas virus-símiles-virus-like particles o VLP) correlaciona bien con la presencia de ADN de VPH en extendidos cervicales. Los anticuerpos invocados reconocen epítopes estructurales tipo-específicos presentes en VLPs, particularmente contra la proteína L1 de la cápside viral. La respuesta humoral (IgG) contra VPH es estable en el tiempo. Se generan anticuerpos neutralizantes, aunque frecuentemente en bajo título, y se consideran protectores contra nueva infección con ese mismo serotipo. La serología basada en VLP ha sido usada como marcador de exposición acumulativa a VPH, y conducta sexual, a pesar que se 
conoce que la seroconversión puede ser tardía o no occurir en un subgrupo de mujeres que poseen ADN de VPH. Globalmente, la frecuencia y el título de diversos tipos de anticuerpos generados contra VPH muestran una gran variabilidad que es dependiente de la especificidad del serotipo de VPH, del epítope reconocido, del tipo de muestra, y de la sensibilidad del ensayo. La mera definición del valor de corte para estos anticuerpos puede entorpecer la comparación entre diferentes vigilancias epidemiológicas. En lo relativo a especificidad de serotipo, se ha demostrado que las VLPs de cada VPH inducen anticuerpos séricos que son genotipo-específicos, con la excepción de los serotipos 6 y 11 que se consideran con reactividad cruzada, y los serotipos 31 y 45 que inducen bajos niveles de anticuerpos cruzados contra VPH 33 y 18, respectivamente. Igualmente, variantes de VPH 16 se considera que pertenecen al mismo serotipo.

\section{Desarrollo de vacunas anti-papilomavirus. Modelos animales}

Desde inicios de los 90s, diferentes grupos han tenido éxito en generar L1 recombinante o VLPs L1 y L2 a partir de todos los papilomavirus conocidos, imitando muy cercanamente a los viriones pero carentes del genoma viral. VLPs producidos en células procariotes (bacterias) y eucariotes (levaduras, insectos, mamíferos) reproducen la antigenicidad de viriones nativos presentando epítopes estructurales, que se ha demostrado, inducen anticuerpos neutralizantes tipo específico. Aunque las VLPs son los candidatos naturales como inmunógenos, diversos otros antígenos, incluyendo proteínas estructurales y no estructurales del papilomavirus han sido testeadas en diferentes protocolos. Además, se han fusionado proteínas tempranas de VPH, tales como E7, E6 y E2 a VLPs. Se ha comprobado que estas vacunas de VLP quiméricas inducen anticuerpos neutralizantes y respuesta citotóxica específica para la proteína temprana en modelos murinos, como se menciona más adelante. Finalmente, se han empleado virus recombinantes, en diferentes vectores, como vacunas de $\mathrm{ADN}$, solas o en combinación con adjuvantes.

Los modelos animales de infección por papilomavirus han sido extraordinariamente valiosos en la investigación y desarrollo de vacunas anti-virales para uso profiláctico y terapéutico. La vacunación con VLPs especie-específicos purificados, induce anticuerpos neutralizantes que protegen contra una exposición al virus vivo en conejos, vacas y perros. Entre estos modelos, la infección de mucosas causada por papilomavirus oral canino (canine oral papillomavirus-
COPV) y papilomavirus oral de conejo (rabbit oral papillomavirus- ROPV) son los que más se asemejan a las infecciones por papilomavirus humano genital. Interesantemente, vacunas basadas en el mismo blanco viral pueden ser efectivas con fines profilácticos o terapéuticos en bovinos, induciendo la regresión de lesiones precoces. La protección es de larga duración, al menos en conejos, en quienes se ha inducido anticuerpos neutralizantes mediante la inyección de CRPV VLPs. En macacos rhesus, la vacunación con una vacuna en base a VLP de VPH 16 L1 induce poderosa inmunidad humoral, incluyendo anticuerpos neutralizantes, y respuesta inmune celular. Aunque ningún papilomavirus se sabe que infecte a los ratones comunes de laboratorio, se dispone de modelos experimentales basados en líneas celulares de ratones transformados con VPH de alto riesgo, capaces de inducir tumores y metástasis. Los modelos en ratones han sido particularmente útiles en testear diferentes vías de administración: la vía intranasal y oral para VLPs de VPH solos, o en combinación con diferentes adyuvantes, han resultado ser immunogénicas. Recientemente se ha comprobado que capsómeros L1 de VPH16, administrados tanto por vía subcutánea como intranasal, inducen potente respuesta de linfocitos citotóxicos, comparable con aquella lograda con VLPs. Se han descrito resultados similares para lesiones en perros inducidas por COPV. Se han utilizado modelos murinos para comprobar que respuesta anti-VPH específica puede obtenerse mediante inmunización con ADN, con lo cual se controla el desarrollo de tumor o de metástasis y promueve la regresión del tumor. En conjunto, los auspiciosos resultados obtenidos con diferentes modelos animales han justificado el desarrollo de vacunas anti-VPH en humanos.

\section{Vacunas para prevenir infecciones por papilomavirus humanos}

Las vacunas para prevenir infecciones por VPH pretenden inducir anticuerpos neutralizantes dirigidos contra epítopes conformacionales de las proteínas de la cápside, los que muy probablemente serán tipo específicos. La protección contra infección depende de la cantidad de anticuerpos producidos por el huésped, su disponibilidad en el sitio de la infección y la persistencia de anticuerpos neutralizantes a lo largo del tiempo. En forma análoga a estudios efectuados en animales, han sido generados y testeados VLPs de los serotipos más prevalentes de VPH como vacunas candidatas existiendo varios estudios clínicos en marcha. La mayoría de estos estudios son randomizados, placebo-control, ciegos, e incluyen mujeres jóvenes 
que viven en diferentes países a lo largo del mundo. Hasta ahora, los estudios publicados con vacuna VPH profiláctica en humanos han demostrado que la vacuna es bien tolerada, genera una enérgica respuesta inmune varias veces superior que aquella lograda en población infectada en forma natural, y previene con alta eficacia tanto la infección como la enfermedad ${ }^{5}$. El primer estudio comprobatorio de principio con una vacuna VLP L1 de VPH 16 demostró que mujeres inyectadas con VLPs de VPH serotipo 16 resultaron ser protegidas contra infección al compararlas con un grupo placebo ${ }^{6}$. Mas aún, durante el seguimiento de 17 meses, los pocos casos de lesión cervical atribuidos a este VPH se desarrollaron sólo en mujeres que no recibieron la vacuna. La eficacia de esta vacuna fue calculada en 90 a $100 \%$. Estos resultados son muy estimulantes y constituyen la factibilidad de emplear esta vacuna monovalente de VPH desarrollada por Merck, Sharp \& Dohme. No obstante, HPV-16 es responsable de sólo cerca del $50 \%$ de todos los cánceres cervicales y, como se mencionara antes, la respuesta inmune contra VPH es tipo específica. En consecuencia, se hicieron esfuerzos para desarrollar vacunas multivalentes que pudieran controlar la mayoría de las infecciones por VPH asociadas con enfermedad cervical, incluyendo los serotipos $-18,-31,-33,-45$, entre otros. Más aún, serotipos de VPH de bajo riesgo son responsables de más del $90 \%$ de las verrugas genitales y papilomatosis laríngea recurrente. Uno de estos estudios consistió en testear la seguridad y eficacia de una vacuna tetravalente (VPH $6,11,16,18$ ) en base a VLPs. El estudio, llevado a cabo en Brasil, E.U.A. y varios países nórdicos, incluyó el seguimiento de más de 1.100 mujeres durante 30 meses, y permitió demostrar que una vacuna tetravalente VPH 6/11/16/18 basada en VLPs L1, producida en levaduras, y que contenía hidróxido de aluminio como adjuvante, inducía altas concentraciones de anticuerpos anti VPH. La respuesta inmune se mantenía aún después de 30 meses de seguimiento y permanecía al mismo nivel o sobre el observado en mujeres infectadas en forma natural ${ }^{7}$. Es importante destacar que esta vacuna tetravalente en base a VLPs de VPH 6, 11, 16 y 18, efectivamente previno la adquisición de infección y enfermedad clínica causada por serotipos comunes de VPH, incluyendo verrugas genitales y neoplasias cervicales intraepiteliales. Está en curso un estudio a gran escala que ha reclutado a 25.000 mujeres jóvenes. Los resultados de estos ensayos de fase III, se espera que estén pronto disponibles, de manera que la vacuna sea comercializada en los años 2006 ó 2007. Futuros estudios se harán vacunando adolescentes, mujeres adultas en edad media y hombres. Un ensayo similar de una vacuna bivalente VLP de VPH (16/18) formulada con AS04 como adjuvante, financiada por Glaxo SmithKline, demonstró que esta vacuna alcanzó una eficacia cercana a $90 \%$ para prevenir las infecciones persistentes y anormalidades cervicales asociadas, en mujeres jóvenes, por un período superior a 27 meses $^{8}$.

\section{Vacunas terapéuticas}

Nuevas estrategias terapéuticas al cáncer cervical incluyen inmunización con proteínas tempranas y péptidos derivados de VPHs de alto riesgo, con la intención de eliminar células epiteliales en el tracto ano-genital que ya está infectado con papilomavirus. La respuesta inmune natural a antígenos codificados en papilomavirus es débil, con la excepción de la proteína E7, a la cual se observa una respuesta inmune humoral en la mayoría de los casos de carcinoma cervical invasor. La respuesta inmune a las proteínas E2 y E6 puede predecir también la regresión de la enfermedad asociada a papilomavirus. Así, está siendo orientada la inmunoterapia hacia algunas de las proteínas tempranas de VPH, particularmente E6 y E7, para el tratamiento de neoplasias inducidas por papilomavirus. Los inmunógenos incluyen péptidos sintéticos, proteínas recombinantes, VLPs quiméricas y vectores virales vivos de expresión. Estudios pre-clínicos en modelos animales indican que algunas de estas vacunas pueden erradicar tumores VPH positivos.

Aunque se ha demostrado que diversas proteínas tempranas de papilomavirus son inmunogénicas en pacientes con cáncer cervical asociado a VPH, se han efectuado ensayos clínicos de vacunas terapéuticas en pacientes con un estado avanzado de enfermedad, en quienes es esperable una pobre respuesta inmune. Pese a todo, se han obtenido algunos resultados favorables y respuesta parcial en pacientes con lesiones intra-epiteliales de alto grado. Se han observado resultados promisorios en pacientes con verrugas genitales. Están en curso ensayos clínicos orientados a demostrar la inmunogenicidad de una vacuna terapéutica en base a ADN para la displasia anal, consistente en un plásmido encapsulado, conteniendo las secuencias de VPH-16 E7 y múltiples epítopes restrictos de HLA-A2. También está siendo estudiado el uso potencial de células dendríticas que portan antígenos de VPH.

Pese a los auspiciosos resultados de diversos estudios en marcha que comprueban buenos perfiles de seguridad e inducción de altos títulos de anticuerpos específicos por vacunas anti-papilomavirus basadas en VLP, hay un sinnúmero de aspectos que requieren atención. Aún si una vacuna demuestra ser efectiva contra algunos serotipos de VPH, se ignora cuánto dura la protección y cuál será el impacto de este tipo de prevención en la historia del cáncer cervical. Se espera 
que una vacuna anti-VPH multivalente reducirá el número de intervenciones tales como colposcopias, biopsias y tratamiento de lesiones precursoras. Sin embargo, los procedimientos de tamizaje basados en el test de Papanicolaou, incluyendo el recientemente aprobado test de ADN de VPH para el cáncer cervical primario en E.U.A., continuarán jugando un papel esencial en la detección de cáncer cervical. Aún más, ignoramos si una cobertura contra algunos genotipos pudiera favorecer la emergencia de serotipos más patogénicos. La existencia de mecanismos tumorales para escapar del sistema inmune sitúa a las vacunas terapéuticas para cánceres asociados a VPH en la misma senda que las vacunas terapéuticas para otros cánceres: hasta ahora, ninguna ha demostrado ser completamente efectiva. El desarrollo de tratamientos multimodales para inducir potentes respuestas inmunes y evitar el escape a tratamientos individuales parece contribuir al control de los precursores de neoplasia cervical del cuello uterino y finalmente al carcinoma invasor.

\section{Referencias}

1.- Muñoz N, Bosch F X, de Sanjose S, Herrero R, Castellsague X, Shah KV, et al. Epidemiologic classification of human papillomavirus types associated with cervical cancer. N Engl J Med 2003; 348: 518-27.

2.- Zur Hausen H. Immortalization of human cells and their malignant conversion by high risk papillomavirus genotypes. Sem Cancer Biol 1999; 9: 405-11.

3.- Munger K, Howley P M. Human papillomavirus immortalization and transformation functions. Virus Res 2002; 89: 213-28.

4.- Konya J, Dillner J. Immunity to oncogenic human papillomaviruses. Adv Cancer Res 2001; 82: 205-38.

5.- Frazer I. Vaccines for papillomavirus infection. Virus Res 2002; 89: 271-4.

6.- Koutsky L A, Ault K A, Wheeler C M, Brown D R, Barr E, Alvarez F B, et al. A controlled trial of a human papillomavirus type 16 vaccine. N Engl J Med. 2002; 347: 1645-51.

7.- Harper D M, Franco E L, Wheeler C M, Ferris D G, Jenkins D, Schuind A, et al.
Efficacy of a bivalent L1 virus-like particle vaccine in prevention of infection of human papillomavirus type 16 and 18 in young women: a randomised controlled trial. Lancet 2004; 364: 1757-65.

9. Villa LL, Costa R L, Petta C A, Andrade R P, Ault K A, Giuliano A R, et al. Prophylactic quadrivalent human papillomavirus (types 6, 11, 16, and 18) L1 virus-like particle vaccine in young women: a randomised double-blind placebo-controlled multicentre phase II efficacy trial. Lancet Oncology 2005; 6: 271-8. 